Astronomy Letters, 2017, Vol. 43, No 4, pp. 241-251

\title{
Refinement of the Parameters of Three Selected Model Galactic Potentials Based on the Velocities of Objects at Distances up to $200 \mathrm{kpc}$
}

\author{
V.V. Bobylev ${ }^{1}$, A.T. Bajkova ${ }^{1}$, and A.O. Gromov ${ }^{2}$ \\ (1) Pulkovo Astronomical Observatory, Russian Academy of Sciences, Pulkovskoe sh. 65, St. \\ Petersburg, 196140 Russia \\ (2) Department of Space Technologies and Applied Astrodynamics, St. Petersburg State \\ University, Universitetskii pr. 35, St. Petersburg, 198504 Russia
}

\begin{abstract}
This paper is a continuation of our recent paper devoted to refining the parameters of three component (bulge, disk, halo) axisymmetric model Galactic gravitational potentials differing by the expression for the dark matter halo using the velocities of distant objects. In all models the bulge and disk potentials are described by the Miyamoto-Nagai expressions. In our previous paper we used the Allen-Santillán (I), Wilkinson-Evans (II), and Navarro-Frenk-White (III) models to describe the halo. In this paper we use a spherical logarithmic Binney potential (model IV), a Plummer sphere (model V), and a Hernquist potential (model VI) to describe the halo. A set of present-day observational data in the range of Galactocentric distances $R$ from 0 to $200 \mathrm{kpc}$ is used to refine the parameters of the listed models, which are employed most commonly at present. The model rotation curves are fitted to the observed velocities by taking into account the constraints on the local matter density $\rho_{\odot}=0.1 M_{\odot} \mathrm{pc}^{-3}$ and the force $K_{z=1.1} / 2 \pi G=77 M_{\odot} \mathrm{pc}^{-2}$ acting perpendicularly to the Galactic plane. The Galactic mass within spheres of radius 50 and $200 \mathrm{kpc}$ are shown to be, respectively, $M_{50}=(0.409 \pm 0.020) \times 10^{12} M_{\odot}$ and $M_{200}=(1.395 \pm 0.082) \times 10^{12} M_{\odot}$ in model IV,$M_{50}=(0.417 \pm 0.034) \times 10^{12} M_{\odot}$ and $M_{200}=(0.469 \pm 0.038) \times 10^{12} M_{\odot}$ in model $\mathrm{V}$, and $M_{50}=(0.417 \pm 0.032) \times 10^{12} M_{\odot}$ and $M_{200}=(0.641 \pm 0.049) \times 10^{12} M_{\odot}$ in model VI. Model VI looks best among the three models considered here from the viewpoint of the achieved accuracy of fitting the model rotation curves to the measurements. This model is close to the Navarro-Frenk-White model III refined and considered best in our previous paper, which is shown using the integration of the orbits of two globular clusters, Lynga 7 and NGC 5053, as an example.
\end{abstract}

\section{INTRODUCTION}

It is necessary to have a reliable model gravitational potential to study the structure of the Galaxy and its dynamical properties or to construct the orbits of Galactic objects. The circular velocities of objects $V_{\text {circ }}$ at various distances $R$ from the Galactic rotation axis (Galactic rotation curve) are among the main sources for the construction of such a model. The accuracy of any model potential depends on the accuracy of determining the distances to the objects used for its construction. 
At present, a high accuracy of distance measurements, on average, $\sim 10 \%$, has been achieved for a number of Galactic objects. In particular, these include more than 100 Galactic masers with measured trigonometric parallaxes, line-of-sight velocities, and proper motions (Reid et al. 2014). Hydrogen clouds at the tangent points also have a high positional accuracy. However, all these objects are no farther than $R=20 \mathrm{kpc}$. The Galactic rotation curve constructed from hydrogen clouds and masers has been used by various authors to refine several model potentials (Irrgang et al. 2013; Bobylev and Bajkova 2013; Reid et al. 2014; Butenko and Khoperskov 2014). This rotation curve is characterized by a fairly large circular velocity of the Galaxy at the solar distance, $V_{\odot} \sim 250 \mathrm{~km} \mathrm{~s}^{-1}$. This leads to an increase in the estimated mass of the Galaxy and its components (disk, halo) compared to that obtained at the standard velocity $V_{\odot}=220 \mathrm{~km} \mathrm{~s}^{-1}$.

Analysis of the observational data at progressively larger $R$ is of great interest. The possibilities for this are continuously expanding. Here is an incomplete list of the most interesting results. Gnedin et al. (2010) found the Galactic mass within a sphere of radius $80 \mathrm{kpc}$ from stars at distances $R<80 \mathrm{kpc}$ to be $M_{80}=0.69_{-1.2}^{+3.0} \times 10^{12} M_{\odot}$. Deason et al. (2012b) found the Galactic mass within a sphere of radius $150 \mathrm{kpc}$ from distant blue horizontal branch giants to be $M_{150}=(0.5-0.1) \times 10^{12} M_{\odot}$. Bhattacharjee et al. (2014) estimated the Galactic mass within a sphere of radius $200 \mathrm{kpc}$ from combined data to be $M_{200} \geq(0.68 \pm 0.41) \times 10^{12} M_{\odot}$. Eadie et al. (2015) found the Galactic mass within a sphere of radius $260 \mathrm{kpc}$ from globular clusters and dwarf galaxies to be $M_{260}=1.37 \times 10^{12} M_{\odot}$. Based on the effect of local Hubble flow deceleration and data on galaxies in the neighborhoods of the Local Group, Karachentsev et al. (2009) estimated the total mass of the Local Group and the ratio of the Galactic and M31 mass to be 4:5. The total mass of the Galaxy obtained by this independent method is $M_{350}=(0.84 \pm 0.09) \times 10^{12} M_{\odot}$.

This article is a continuation of the paper by Bajkova and Bobylev (2016) devoted to refining the parameters of model Galactic gravitational potentials, where three-component (bulge, disk, halo) models differing by the expression for the dark matter halo were considered. The expressions from Miyamoto and Nagai (1975) were traditionally used to describe the bulge and the disk, and the following three models were considered to describe the halo: the Allen-Santillán (I), Wilkinson-Evans (II), and Navarro-Frenk-White (III) models.

The goal of this paper is to refine the parameters of the Galactic gravitational potential for other models of the dark matter halo. For this purpose, we use three models where the halo is represented by a logarithmic Binney potential (Binney 1981; Evans 1993; Fellhauer et al. 2006) (model IV), a Plummer (1911) sphere (model V), and a Hernquist (1990) potential (model VI). Here, as in our previous paper (Bajkova and Bobylev 2016), we use observational data in the range of distances $0-200 \mathrm{kpc}$ and constraints on the local matter density $\rho_{\odot}$ and the force $K_{z=1.1}$. The selected models are employed most commonly at present, because they are realistic and mathematically simple and their analytic expressions are closed. Therefore, their correction based on new data is an important task.

\section{DATA}

Since this is a continuation of our previous paper (Bajkova and Bobylev 2016), we use exactly the same data. First, these include the line-of-sight velocities of HI clouds at the tangent points from Burton and Gordon (1978). These data on the rotation curve fill the range of 
distances $R<4 \mathrm{kpc}$. Second, these include a sample of masers with measured trigonometric parallaxes, proper motions, and line-of-sight velocities. They are located in the range of distances $R$ from 4 to $20 \mathrm{kpc}$. Third, these include the average circular rotation velocities from Bhattacharjee et al. (2014) calculated using objects at distances $R$ from 20 to $\approx 200$ kpc. The velocities of 1457 blue horizontal branch giants, $2227 \mathrm{~K}$ giants, 16 globular clusters, 28 distant halo giants, and 21 dwarf galaxies were used for this purpose. Since Bhattacharjee et al. (2014) constructed the Galactic rotation curve with $R_{\odot}=8.3 \mathrm{kpc}$ and $V_{\odot}=244 \mathrm{~km}$ $\mathrm{s}^{-1}$, we also calculate the circular velocities of objects with these parameters.

The following can be said in more detail about the masers. The VLBI measurements of 103 masers are described in the general review of Reid et al. (2014). Subsequently, these authors published improved values of the measured parameters for some of the masers $(\mathrm{Wu}$ et al. 2014; Choi et al. 2014; Sato et al. 2014; Hachisuka et al. 2015). After the addition of the most recent published VLBI measurements (Motogi et al. 2015; Burns et al. 2015), we obtained a sample with data on 130 sources. We do not include the masers located at distances $R<4 \mathrm{kpc}$ in our sample, because their velocities are distorted by the central Galactic bar.

As a result, we rely on the line-of-sight velocities of hydrogen clouds at the tangent points and the data on 130 masers with measured trigonometric parallaxes at distances $R$ less than $25 \mathrm{kpc}$, and the rotation curve from Bhattacharjee et al. (2014) serves us as the data at greater distances.

\section{MODEL GALACTIC POTENTIALS}

\section{Introductory Mathematical Expressions}

In all of the models being considered here the axisymmetric Galactic potential is represented as a sum of three components - a central spherical bulge $\Phi_{b}(r(R, z))$, a disk $\Phi_{d}(r(R, z))$, and a massive spherical dark matter halo $\Phi_{h}(r(R, z))$ :

$$
\Phi(R, z)=\Phi_{b}(r(R, z))+\Phi_{d}(r(R, z))+\Phi_{h}(r(R, z)) .
$$

Here, we use a cylindrical coordinate system $(R, \psi, z)$ with the coordinate origin at the Galactic center. In a rectangular coordinate system $(x, y, z)$ with the coordinate origin at the Galactic center the distance to a star (spherical radius) will be $r^{2}=x^{2}+y^{2}+z^{2}=R^{2}+z^{2}$.

In accordance with the convention adopted in Allen and Santillán (1991), we express the gravitational potential in units of $100 \mathrm{~km}^{2} \mathrm{~s}^{-2}$, the distances in $\mathrm{kpc}$, and the masses in units of the Galactic mass $M_{\text {gal }}=2.325 \times 10^{7} M_{\odot}$, corresponding to the gravitational constant $G=1$.

The expression for the mass density follows from the Poisson equation

$$
4 \pi G \rho(R, z)=\nabla^{2} \Phi(R, z)
$$

and is

$$
\rho(R, z)=\frac{1}{4 \pi G}\left(\frac{d^{2} \Phi(R, z)}{d R^{2}}+\frac{1}{R} \frac{d \Phi(R, z)}{d R}+\frac{d^{2} \Phi(R, z)}{d z^{2}}\right) .
$$

The force acting in the $z$ direction perpendicular to the Galactic plane is defined as

$$
K_{z}(z, R)=-\frac{d \Phi(z, R)}{d z} .
$$


We will need Eqs. (3) and (4) below to solve the problem of fitting the parameters of the model gravitational potentials with the constraints imposed on the local dynamical matter density $\rho_{\odot}$ and the force $K_{z}\left(z, R_{\odot}\right)$ at $z=1.1 \mathrm{kpc}$ that are known from observations.

In addition, we will need the expressions to calculate:

(1) the circular velocities

$$
V_{\text {circ }}(R)=\sqrt{R \frac{d \Phi(R, 0)}{d R},}
$$

(2) the Galactic mass contained in a sphere of radius $r$ based on the spherically symmetric model

$$
M(r)=r^{2} \frac{d \Phi(r)}{d r}
$$

(3) the parabolic velocity or the escape velocity of a star from the attractive Galactic field

$$
V_{e s c}(R, z)=\sqrt{-2 \Phi(R, z)}
$$

(4) the Oort parameters

$$
\begin{aligned}
A & =\frac{1}{2} \Omega_{\odot}^{\prime} R_{\odot}, \\
B & =\Omega_{\odot}+A,
\end{aligned}
$$

where $\Omega=V / R$ is the angular velocity of Galactic rotation $\left(\Omega_{\odot}=V_{\odot} / R_{\odot}\right), \Omega_{\odot}^{\prime}$ is the first derivative of the angular velocity with respect to $R$ and $R_{\odot}$ is the Galactocentric distance of the Sun;

(5) the surface density of gravitating matter within $z_{\text {out }}$ of the Galactic $z=0$ plane

$$
\Sigma_{\text {out }}\left(z_{\text {out }}\right)=2 \int_{0}^{z_{\text {out }}} \rho(R, z) d z=\frac{K_{z}}{2 \pi G}+\frac{2 z_{\text {out }}\left(B^{2}-A^{2}\right)}{2 \pi G},
$$

Note that the spherically symmetric model describes satisfactorily the density distribution in a galaxy only when the disk mass is much smaller than the mass of its spherical components. This condition is fulfilled in the case of our Galaxy. However, even if this condition is not fulfilled completely, Eq. (6) is well suited for a rough estimation of the total mass within a radius $r$. Generally, the mass found within the spherically symmetric model will be an upper limit: $M(r) \leq r^{2} d \Phi(r) / d r$ (Zasov and Postnov 2006).

\section{Bulge and Disk}

In all of the models being considered here the bulge, $\Phi_{b}(r)$ and disk, $\Phi_{d}(r(R, z))$ potentials are represented by the expressions from Miyamoto and Nagai (1975):

$$
\begin{gathered}
\Phi_{b}(r)=-\frac{M_{b}}{\left(r^{2}+b_{b}^{2}\right)^{1 / 2}}, \\
\Phi_{d}(R, z)=-\frac{M_{d}}{\left\{R^{2}+\left[a_{d}+\left(z^{2}+b_{d}^{2}\right)^{1 / 2}\right]^{2}\right\}^{1 / 2}},
\end{gathered}
$$

where $M_{b}$ and $M_{d}$ are the masses of the components, $b_{b}, a_{d}$, and $b_{d}$ are the scale lengths of the components in kpc. The corresponding expressions for the mass densities $\rho_{b}(R, z)$ and $\rho_{d}(R, z)$ are

$$
\rho_{b}(r)=\frac{3 b_{b}^{2} M_{b}}{4 \pi G\left(r^{2}+b_{b}^{2}\right)^{5 / 2}}
$$




$$
\rho_{d}(R, z)=\frac{b_{d}^{2} M_{d}}{4 \pi G\left(z^{2}+b_{d}^{2}\right)^{3 / 2}} \frac{a_{d} R^{2}+\left(a_{d}+3 \sqrt{z^{2}+b_{d}^{2}}\right)\left(a_{d}+\sqrt{z^{2}+b_{d}^{2}}\right)^{2}}{\left(R^{2}+\left(a_{d}+\sqrt{z^{2}+b_{d}^{2}}\right)^{2}\right)^{5 / 2}} .
$$

Expressions (11) and (13) describe a Plummer (1911) sphere, while relations (12) and (14) describe a generalized Kuzmin (1956) disk.

Integrating the mass densities over the entire volume of the Galaxy gives the expected bulge and disk masses: $m_{b}=M_{b}$, and $m_{d}=M_{d}$. The bulge and disk contributions to the circular velocity are, respectively,

$$
\begin{gathered}
V_{\text {circ }(b)}^{2}(R)=\frac{M_{b} R^{2}}{\left(R^{2}+b_{b}^{2}\right)^{3 / 2}}, \\
V_{c i r c(d)}^{2}(R)=\frac{M_{d} R^{2}}{\left(R^{2}+\left(a_{d}+b_{d}\right)^{2}\right)^{3 / 2}} .
\end{gathered}
$$

The corresponding expressions for $K_{z}^{b}(z, R)$ and $K_{z}^{d}(z, R)$ are

$$
\begin{gathered}
K_{z}^{b}(z, R)=\frac{z M_{b}}{\left(R^{2}+z^{2}+b_{b}^{2}\right)^{3 / 2}}, \\
K_{z}^{d}(z, R)=\frac{z M_{d}\left(a_{d}+\sqrt{z^{2}+b_{d}^{2}}\right)}{\sqrt{z^{2}+b_{d}^{2}}\left(R^{2}+\left(a_{d}+\sqrt{z^{2}+b_{d}^{2}}\right)^{2}\right)^{3 / 2}} .
\end{gathered}
$$

\section{Dark Matter Halo}

Model IV. The halo component is represented by a logarithmic potential in the form proposed by Binney (1981):

$$
\Phi_{h}(R, z)=-\frac{v_{0}^{2}}{2} \ln \left(R^{2}+a_{h}^{2}+\frac{z^{2}}{q_{\Phi}^{2}}\right),
$$

where $v_{0}$ is the velocity in $\mathrm{km} \mathrm{s}^{-1}, q_{\Phi}$ is the axial ratio of the ellipsoid: $q_{\Phi}=1$ for a spherical halo, $q_{\Phi}<1$ for an oblate one, and $q_{\Phi}>1$ for a prolate one. We take $q_{\Phi}=1$. In this case, the mass density is

$$
\rho_{h}(r)=\frac{v_{0}^{2}}{4 \pi G} \frac{\left(3 a_{h}^{2}+r^{2}\right)}{\left(r^{2}+a_{h}^{2}\right)^{2}}
$$

It is easy to show that integrating the mass density over a sphere of radius $r$ gives a halo mass that depends almost linearly on $r$. The contribution to the circular velocity is

$$
V_{\text {circ }(h)}^{2}=\frac{v_{0}^{2} R^{2}}{R^{2}+a_{h}^{2}} .
$$

The expression for $K_{z}^{h}(z, R)$ is

$$
K_{z}^{h}(z, R)=\frac{z v_{0}^{2}}{R^{2}+z^{2}+a_{h}^{2}} .
$$

In the three-component model Galactic potential of Fellhauer et al. (2006) the Hernquist (1990) potential was used to describe the bulge, the disk was described by Eq. (12), and 
the halo was described by the logarithmic Binney (1981) potential (19). In the original form this approach has been actively used in recent years to construct the Galactic orbits of various objects (see, e.g., Ružička et al. 2010; Gontcharov et al. 2011; Veras and Evans 2013; Dremova et al. 2015).

Model V. In this model we use a Plummer (1911) sphere (coincident with Eq. (11)) to describe the halo potential. As a result, we have

$$
\Phi_{h}(r)=-\frac{M_{h}}{\left(r^{2}+a_{h}^{2}\right)^{1 / 2}} .
$$

The mass density is

$$
\rho_{h}(r)=\frac{3 a_{h}^{2} M_{h}}{4 \pi G\left(r^{2}+a_{h}^{2}\right)^{5 / 2}} .
$$

Integrating the mass density over the entire volume of the Galaxy gives the mass $m_{h}=M_{h}$. The contribution to the circular velocity is

$$
V_{\text {circ }(h)}^{2}(R)=\frac{M_{h} R^{2}}{\left(R^{2}+a_{h}^{2}\right)^{3 / 2}} .
$$

The expression for $K_{z}^{h}(z, R)$ is

$$
K_{z}^{h}(z, R)=\frac{z M_{h}}{\left(R^{2}+z^{2}+a_{h}^{2}\right)^{3 / 2}} .
$$

Such a three-component model Galactic potential (a bulge in the form (11), a disk in the form (12), and a halo in the form (23)) was first proposed by Dauphole and Colin (1995). Therefore, we call this approach the Dauphole-Colin model, and this approach is much in demand at present. For example, it was used by Kalirai et al. (2007) to study the orbit of the globular cluster NGC 6397, by Just et al. (2009) to construct the Galactic orbits of open clusters, and by Bailer-Jones (2015) to study close stellar encounters.

Model VI. The halo component is represented by the Hernquist (1990) potential

$$
\Phi_{h}(r)=-\frac{M_{h}}{r+a_{h}}
$$

The mass density is

$$
\rho_{h}(r)=\frac{a_{h} M_{h}}{2 \pi G r\left(r+a_{h}\right)^{3}} .
$$

Integrating the mass density over the entire volume of the Galaxy gives the mass $m_{h}=M_{h}$. The contribution to the circular velocity is

$$
V_{\text {circ }(h)}^{2}(R)=\frac{M_{h} R}{\left(R+a_{h}\right)^{2}} .
$$

The expression for $K_{z}^{h}(z, r)$ is

$$
K_{z}^{h}(z, r)=\frac{z M_{h}}{r\left(r+a_{h}\right)^{2}}
$$

The potential (27) is commonly and widely used to describe the central spherical bulge (Fellhauer et al. 2006). However, this potential is occasionally used to describe the halo or the bulge-halo pair (see, e.g., Eadie et al. 2015; Capuzzo-Dolcetta and Fragione 2015). 


\section{Parameter Fitting}

The parameters of the model potentials are found by least-squares fitting to the measured rotation velocities of Galactic objects. We use the unit weights, because they provide the smallest absolute residual between the data and the model rotation curve. In addition, we used (Irrgang et al. 2013) the constraints on (1) the local dynamical matter density $\rho_{\odot}=0.1 M_{\odot} \mathrm{pc}^{-3}$ and $(2)$ the force acting perpendicularly to the Galactic plane or, more specifically, $K_{z=1.1} / 2 G=77 M_{\odot} \mathrm{pc}^{-2}$.

The local dynamical matter density $\rho_{\odot}$, which is the sum of the bulge, disk, and dark matter densities in a small solar neighborhood, together with the surface density $\Sigma_{1.1}$ are the most important additional constraints in the problem of fitting the parameters of the model potentials to the measured circular velocities (Irrgang et al. 2013):

$$
\begin{gathered}
\rho_{\odot}=\rho_{b}\left(R_{\odot}\right)+\rho_{d}\left(R_{\odot}\right)+\rho_{h}\left(R_{\odot}\right) \\
\Sigma_{1.1}=\int_{-1.1 k p c}^{1.1 k p c}\left(\rho_{b}\left(R_{\odot}, z\right)+\rho_{d}\left(R_{\odot}, z\right)+\rho_{h}\left(R_{\odot}, z\right)\right) d z .
\end{gathered}
$$

The surface density is closely related to the force $K_{z}(z, R)$ in accordance with Eq. (10). Since

the two most important parameters $\rho_{\odot}$ and $K_{z} / 2 \pi G$ are known from observations with a sufficiently high accuracy, introducing additional constraints on these two parameters allows the parameters of the gravitational potential to be refined significantly.

As a result, the parameter fitting problem was reduced to minimizing the following quadratic functional $F$ :

$$
\min F=\sum_{i=1}^{N}\left(V_{\text {circ }}\left(R_{i}\right)-\widetilde{V}_{\text {circ }}\left(R_{i}\right)\right)^{2}+\alpha_{1}\left(\rho_{\odot}-\widetilde{\rho}_{\odot}\right)^{2}+\alpha_{2}\left(K_{z=1.1} / 2 \pi G-\widetilde{K}_{z=1.1} / 2 \pi G\right)^{2}
$$

where the measured quantities are denoted by the tilde, $R_{i}$ are the distances of the objects with measured circular velocities, $\alpha_{1}$ and $\alpha_{2}$ are the weight factors at the additional constraints that were chosen so as to minimize the residual between the data and the model rotation curve provided that the additional constraints hold with an accuracy of at least $5 \%$. Based on the constructed models, we calculated the local surface density of the entire matter $\rho_{\odot}$ and $K_{z=1.1} / 2 \pi G$ related to $\Sigma_{1.1}$ and $\Sigma_{\text {out }}$.

\section{RESULTS AND DISCUSSION}

Table 1 provides the values of the seven sought for parameters $\left(M_{b}, M_{d}, M_{h}, b_{b}, a_{d}, a_{b}, a_{h}\right)$ found by solving the fitting problem for the three model Galactic potentials under consideration and the masses of the Galactic components $m_{b}, m_{d}$, and $m_{h}$. The parameters $M_{b}, M_{d}$, and $M_{h}$ are given in units of the Galactic mass $M_{g}=2.325 \times 10^{7} M_{\odot} ; v_{0}^{2} / 2$ in $\mathrm{km}^{2}$ s-2 is given instead of $M_{h}$ for model IV (see the footnote to the table). The masses of the Galactic components $m_{b}, m_{d}, m_{h}$ are given in units of the solar mass. As has already been said above, the halo mass for models V and VI when integrated over the entire Galaxy is $m_{h}=M_{h}$. The halo mass within a sphere of radius $200 \mathrm{kpc}$ determined by the object velocity measurements is provided for model IV, because the halo mass increases with increasing radius of the sphere $r$ as a linear function of $r$. The last row in the table 


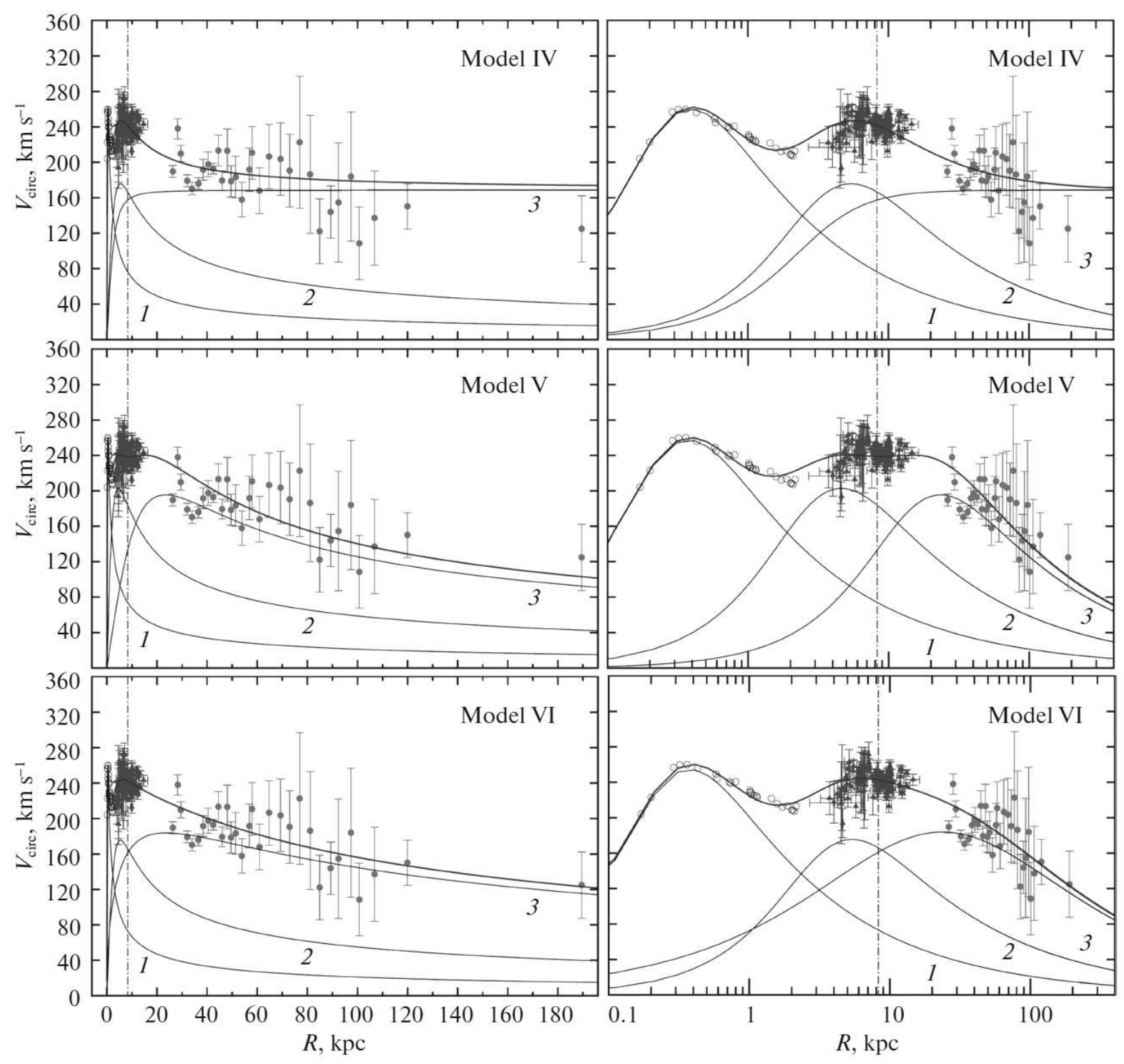

Рис. 1: Model Galactic rotation curves $V_{\text {circ }}(R)$ for potentials IV, V, and VI in the linear (left) and logarithmic (right) distance scales as well as the measured rotation velocities of objects $\widetilde{V}_{\text {circ }}\left(R_{i}\right)$; the vertical dash-dotted line marks the Sun's position, numbers 1, 2, and 3 denote the bulge, disk, and halo contributions, respectively; the open circles, filled triangles, and filled circles indicate the HI velocities, the velocities of masers with measured trigonometric parallaxes, and the velocities from Bhattacharjee et al. (2014), respectively. 
Таблица 1: The model parameters found by fitting to the data

\begin{tabular}{|c|c|c|c|}
\hline Parameters & model IV & model V & model VI \\
\hline$M_{b}\left(M_{g}\right)$ & $486 \pm 10$ & $456 \pm 40$ & $461 \pm 22$ \\
$M_{d}\left(M_{g}\right)$ & $3079 \pm 23$ & $3468 \pm 71$ & $2950 \pm 33$ \\
$M_{h}\left(M_{g}\right)$ & $14210 \pm 858\left(^{*}\right)$ & $16438 \pm 1886$ & $29677 \pm 2791$ \\
$m_{b}\left(10^{9} M_{\odot}\right)$ & $11.30 \pm 0.23$ & $10.60 \pm 0.93$ & $10.71 \pm 0.51$ \\
$m_{d}\left(10^{10} M_{\odot}\right)$ & $7.16 \pm 0.05$ & $8.06 \pm 0.16$ & $6.86 \pm 0.07$ \\
$m_{h}\left(10^{11} M_{\odot}\right)$ & $13.12 \pm 0.20\left(^{* *}\right)$ & $3.81 \pm 0.44$ & $6.88 \pm 0.65$ \\
$b_{b}(\mathrm{kpc})$ & $0.2769 \pm 0.0052$ & $0.2647 \pm 0.0057$ & $0.2720 \pm 0.0130$ \\
$a_{d}(\mathrm{kpc})$ & $3.54 \pm 0.06$ & $2.94 \pm 0.076$ & $3.85 \pm 0.08$ \\
$b_{d}(\mathrm{kpc})$ & $0.2997 \pm 0.0023$ & $0.3126 \pm 0.0022$ & $0.3090 \pm 0.0010$ \\
$a_{h}(\mathrm{kpc})$ & $3.20 \pm 0.45$ & $16.57 \pm 1.38$ & $21.27 \pm 1.06$ \\
\hline$\delta\left(\mathrm{km} \mathrm{s}^{-1}\right)$ & -29.11 & -29.72 & -24.96 \\
\hline Residual noise entropy $E$ & 15.04 & 14.89 & 13.23 \\
\hline
\end{tabular}

The Galactic mass unit is $M_{g}=2.325 \times 10^{7} M_{\odot},\left(^{*}\right) v_{0}^{2} / 2$ in $\mathrm{km}^{2} \mathrm{~s}^{-2}$ is given here, $\left(^{* *}\right)$ the halo mass within a radius of $200 \mathrm{kpc}$ is given here.

gives the residual between the model rotation curve and the measured velocities (data) $\delta=\sqrt{\left(\sum_{i=1}^{N}\left(V_{\text {circ }}\left(R_{i}\right)-\widetilde{V}_{\text {circ }}\left(R_{i}\right)\right)^{2}\right) / N}$. The next-to-last row provides the entropy of the difference between the data and the model rotation curve. It gives an idea of the degree of uniformity of the residual noise is: $E=-\frac{1}{N} \sum_{i=1}^{N}\left|\Delta_{i}\right| \ln \left(\left|\Delta_{i}\right|\right)$, where the residual noise is $\Delta_{i}=V_{\text {circ }}\left(R_{i}\right)-\widetilde{V}_{\text {circ }}\left(R_{i}\right)$. The higher the entropy, the more uniform the noise and, consequently, the better the parameter fitting. As we see, model VI provides the smallest residual $\delta=13.23 \mathrm{~km} \mathrm{~s}^{-1}$ and the greatest entropy of the residual noise $E=-24.96$, i.e., the greatest degree of its uniformity. This model is close to the Navarro-Frenk-White (1997) model III, which we deem best from the viewpoint of the accuracy of fitting to the data. In this case, $E=-24.51$ and $\delta=13.1 \mathrm{~km} \mathrm{~s}^{-1}$ (see Table 1 in Bajkova and Bobylev (2016)).

Table 2 lists the physical quantities calculated from the derived parameters of the model potentials. These include the local disk density $\left(\rho_{\odot}\right)_{d}$, the local dark matter density $\left(\rho_{\odot}\right)_{h}$, the local density of the entire matter $\rho_{\odot}, K_{z=1.1} / 2 G$, the local surface density $\Sigma_{1.1}$ and $\Sigma_{\text {out }}$, the two escape velocities from the Galaxy $V_{\text {esc }}$ for $R=R_{\odot}$ and $R=200 \mathrm{kpc}$, the linear circular rotation velocity of the Sun $V_{\odot}$, , the Oort constants $A$ and $B$, and the Galactic mass $M_{G}$ for four radii of the enclosing sphere.

The errors of all parameters and physical quantities in Tables 1 and 2, respectively, were determined through Monte Carlo simulations using each time 100 independent realizations of random velocity measurement errors by assuming the errors to obey a normal distribution with zero mean and a fixed rms deviation.

The model Galactic rotation curves constructed for potentials IV, V, and VI are presented in Fig. 1. The bulge, disk, and halo contributions are given for each model.

In model IV, in accordance with (21), the circular velocity of the halo increases monotonically with Galactocentric distance (see Fig. 1). In this model it is apparently desirable to artificially correct the halo density function at great distances $(R>200 \mathrm{kpc})$, as is done in model I (Bajkova and Bobylev 2016).

Model $\mathrm{V}$ has the largest disk mass $\left(M_{d}\right)$ compared to our other models, as can be seen 
Таблица 2: The quantities calculated from the parameters of the model potentials found

\begin{tabular}{|c|c|c|c|}
\hline Parameters & model IV & model V & model VI \\
\hline$\left(\rho_{\odot}\right)_{d}\left(M_{\odot} \mathrm{pc}^{-3}\right)$ & $0.092 \pm 0.009$ & $0.089 \pm 0.010$ & $0.090 \pm 0.010$ \\
\hline$\left(\rho_{\odot}\right)_{h}\left(M_{\odot} \mathrm{pc}^{-3}\right)$ & $0.008 \pm 0.001$ & $0.011 \pm 0.001$ & $0.011 \pm 0.001$ \\
\hline$\rho_{\odot}\left(M_{\odot} \mathrm{pc}^{-3}\right)$ & $0.100 \pm 0.010$ & $0.100 \pm 0.010$ & $0.100 \pm 0.010$ \\
\hline$K_{z=1.1} / 2 \pi G\left(M_{\odot} \mathrm{pc}^{-2}\right)$ & $77.0 \pm 6.3$ & $77.1 \pm 6.6$ & $77.2 \pm 5.8$ \\
\hline$\Sigma_{1.1}\left(M_{\odot} \mathrm{pc}^{-2}\right)$ & $71.4 \pm 6.4$ & $78.6 \pm 7.9$ & $76.9 \pm 6.4$ \\
\hline$\Sigma_{\text {out }}\left(M_{\odot} \mathrm{pc}^{-2}\right)$ & $45.2 \pm 7.1$ & $75.0 \pm 14.2$ & $68.9 \pm 10.1$ \\
\hline$V_{e s c, R}=R_{\odot}\left(\mathrm{km} \mathrm{s}^{-1}\right)$ & $450.2 \pm 8.6$ & $516.0 \pm 21.4$ & $524.8 \pm 18.2$ \\
\hline$V_{e s c,} R=200 \mathrm{kpc}\left(\mathrm{km} \mathrm{s}^{-1}\right)$ & $550.7 \pm 16.7$ & $142.5 \pm 5.7$ & $173.9 \pm 6.8$ \\
\hline$V_{\odot}\left(\mathrm{km} \mathrm{s}^{-1}\right)$ & $241.3 \pm 3.9$ & $238.8 \pm 9.4$ & $243.1 \pm 6.8$ \\
\hline$A\left(\mathrm{~km} \mathrm{~s}^{-1} \mathrm{kpc}^{-1}\right)$ & $16.10 \pm 0.62$ & $14.49 \pm 0.60$ & $15.05 \pm 0.52$ \\
\hline$B\left(\mathrm{~km} \mathrm{~s}^{-1} \mathrm{kpc}^{-1}\right)$ & $-12.97 \pm 0.69$ & $-14.27 \pm 1.15$ & $-14.24 \pm 0.84$ \\
\hline$M_{G_{(R \leq 50 k p c)}}\left(10^{12} M_{\odot}\right)$ & $0.409 \pm 0.020$ & $0.417 \pm 0.034$ & $0.417 \pm 0.032$ \\
\hline$M_{G_{(R \leq 100 k p c)}}\left(10^{12} M_{\odot}\right)$ & $0.738 \pm 0.040$ & $0.457 \pm 0.037$ & $0.547 \pm 0.042$ \\
\hline$M_{G_{(R \leq 150 k p c)}}\left(10^{12} M_{\odot}\right)$ & $1.066 \pm 0.061$ & $0.466 \pm 0.037$ & $0.607 \pm 0.047$ \\
\hline$M_{G_{(R \leq 200 k p c)}}\left(10^{12} M_{\odot}\right)$ & $1.395 \pm 0.082$ & $0.469 \pm 0.038$ & $0.641 \pm 0.049$ \\
\hline
\end{tabular}

Таблица 3: The characteristics of the Galactic orbits of the two selected globular clusters calculated using models III and VI

\begin{tabular}{|l|c|c|c|c|}
\hline Cluster & model & $\left\langle a_{\min }\right\rangle, \mathrm{kpc}$ & $\left\langle a_{\max }\right\rangle, \mathrm{kpc}$ & $\langle e\rangle$ \\
\hline Lynga 7 & III & 4.34 & 8.00 & 0.30 \\
& VI & 4.34 & 8.08 & 0.30 \\
\hline NGC 5053 & III & 14.79 & 61.09 & 0.61 \\
& VI & 14.80 & 57.93 & 0.59 \\
\hline
\end{tabular}

from Table 1. It follows from the last rows in Table 2 that based on this model, we obtain the smallest Galactic mass $\left(M_{G}\right)$ among the other models. Models V and VI are attractive in that both the circular velocity of the halo and the overall rotation curve at distances greater than $100 \mathrm{kpc}$ fall off gently. Therefore, there is no need to artificially correct the halo density function.

Note that we took the values justified by Irrgang et al. (2013) as the target parameters in fitting $\rho_{\odot}$ and $K_{z=1.1} / 2 \pi G$. Fitting these parameters basically led to fitting the function $\rho_{\odot}(z)$ and, consequently, the surface density $\Sigma_{1.1}$. The results of fitting to the function $\rho_{\odot}(z)$ obtained by Irrgang et al. (2013) and considered in this paper as a template are shown in Fig. 2. As can be seen from the figure, all of the derived profiles are quite close to one another, but model VI reproduces the required function $\rho_{\odot}(z)$ with the highest accuracy.

Such local parameters of the rotation curve as the velocity $V_{\odot}$ and the Oort constants $A$ and $B$ are well reproduced by the models considered. In model $\mathrm{V}$, however, $B$ is comparable in absolute value to $A$. Therefore, the rotation velocity in a small segment near the Sun is nearly flat $\left(V_{\text {circ }}=\right.$ const $)$, as can be clearly seen from Fig. 1.

Interestingly, the escape velocity $V_{\text {esc }}(R=200 \mathrm{kpc})$ is usually approximately half that at $R=R_{\odot}$. However, for model IV the parabolic velocity at $R=200$ kpc exceeds its value 


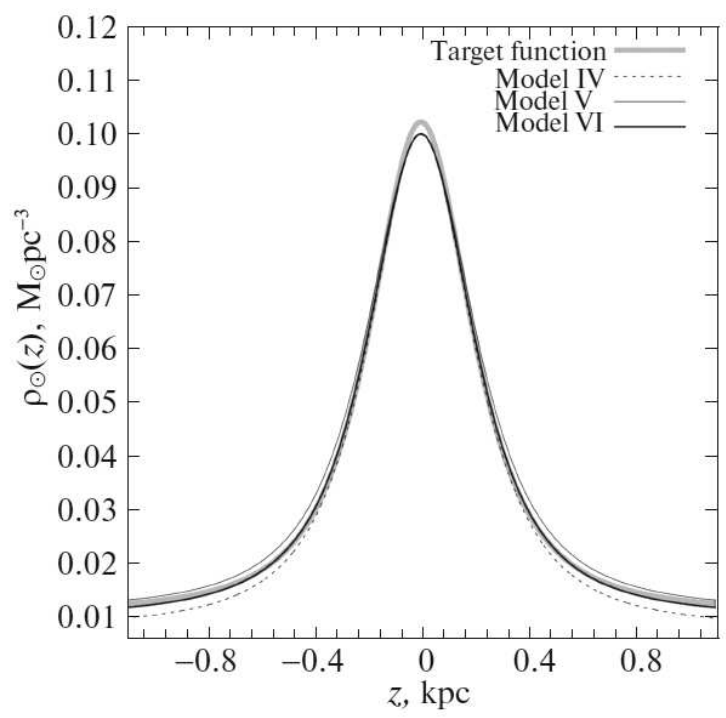

Рис. 2: Results of fitting $\rho_{\odot}(z)$ for models IV, V, and VI. The functions from Irrgang et al. (2013) are considered as the target functions $\rho_{\odot}(z)$.

calculated for $R=R_{\odot}$.

The Galactic mass within a sphere of radius $50 \mathrm{kpc}, M_{50} \approx 0.4 \times 10^{12} M_{\odot}$, that we found based on various model potentials is in good agreement with the results of other authors. For example, Deason et al. (2012a) estimated $M_{50}=(0.42 \pm 0.04) \times 10^{12} M_{\odot}$, from horizontal branch giants; Williams and Evans $(2015)$ found $M_{50}=(0.45 \pm 0.15) \times 10^{12} M_{\odot}$ from horizontal branch giants from the SDSS (Sloan Digital Sky Survey) catalogue.

It is important to note that the Galactic mass estimates that we obtained in this paper based on model VI (the best one in this paper) are close to those based on model III (the best one in Bajkova and Bobylev (2016)) and, consequently, are in good agreement with the results of other authors that are displayed in Fig. 4 from Bajkova and Bobylev (2016).

For an even subtler comparison of models III and VI we integrated the orbits of two globular clusters (Lynga 7 and NGC 5053) from the catalog by Kharchenko et al. (2013) based on the equations of motion given in the Appendix to Irrgang et al. (2013). Figure 3 shows the Galactic orbits of the globular clusters Lynga 7 and NGC 5053 obtained by integration over 500 Myr and 5 Gyr into the past, respectively. Table 3 gives the main characteristics of boxy orbits: the average values of the minimum, $\left\langle a_{\min }\right\rangle$, and maximum, $\left\langle a_{\max }\right\rangle$, distances and the eccentricity $\langle e\rangle$. We can see good agreement between the trajectories both for the cluster close to the Galactic center and the short integration time interval (Lynga 7) and for the fairly distant cluster and the long integration time (NGC 5053).

It is interesting to compare our results with the result of other authors. Although the present-day data suggest that the solar velocity is $V_{\odot}=240 \mathrm{~km} \mathrm{~s}^{-1}$ and the solar Galactocentric distance is $R_{\odot}=8.3 \mathrm{kpc}$, many of the model potentials were constructed for other values of $V_{\odot}$ and $R_{\odot}$. As an example, let us compare our results on the mass decomposition with the results from Dauphole and Colin (1995). In this paper the model potential $\mathrm{V}$ was used, and its parameters at $R_{\odot}=8.0 \mathrm{kpc}$ and velocity $V_{\odot}=225 \mathrm{~km} \mathrm{~s}^{-1}$ were found from the velocities of globular clusters in the range of Galactocentric distances from 0 to $16 \mathrm{kpc}$. The following results were obtained: $m_{b}=13.9 \times 10^{9} M_{\odot}, m_{d}=7.9 \times 10^{10} M_{\odot}, m_{h}=$ $7.91 \times 10^{11} M_{\odot}$. The local mass density was $\rho_{\odot}=0.143 M_{\odot} \mathrm{pc}^{-3}$; the vertical force was 

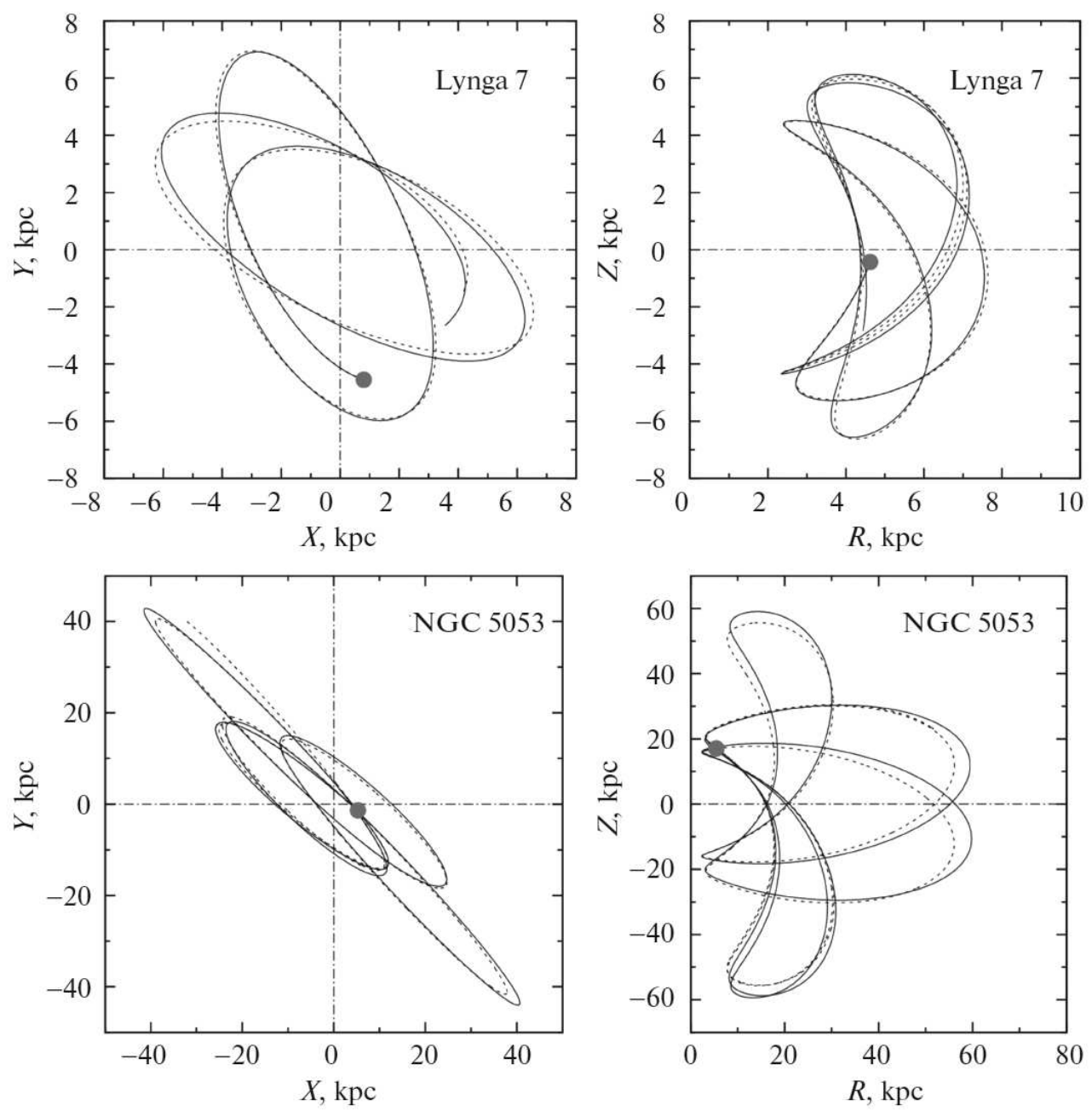

Рис. 3: The Galactic orbits of the globular clusters Lynga 7 (top) and NGC 5053 (bottom) constructed using models III (solid line) and VI (dotted line); the gray circle marks the current position of the cluster.

$K_{z=1.1} / 2 \pi G=84 M_{\odot} \mathrm{pc}^{-2}$, which differs tangibly from the present-day estimates that we used to fit the parameters. By comparing the masses of the components with those presented in Table 1, we can see that the bulge and disk mass estimates are close to our estimates, while the halo masses differ approximately by a factor of 2 . This difference is explained by the fact that the parameters were fitted using different data. Our data on the velocities cover a wide range of distances up to $200 \mathrm{kpc}$, while those from Dauphole and Colin (1995) cover a range only up to $16 \mathrm{kpc}$, with our data showing a considerable decrease in the circular velocities with increasing distance $R$ at great distances. If we extend the model rotation curve from Dauphole and Colin (1995) to large $R$, then we will not get the agreement with our data that is provided by our model rotation curve. All of this suggests how important it is to use the data in a wide range of Galactocentric distances to refine the model Galactic potential. 


\section{CONCLUSIONS}

We considered three three-component (bulge, disk, halo) model Galactic potentials differing by the expression for the halo potential. The observational data spanning the range of Galactocentric distances $R$ from 0 to $\sim 200 \mathrm{kpc}$ were used to refine the parameters of these models. We relied on the line-of-sight velocities of hydrogen clouds at the tangent points and the data on 130 masers with measured trigonometric parallaxes up to distances of about $20 \mathrm{kpc}$, and the average rotation velocities from the review by Bhattacharjee et al. (2014) served us as the data at greater distances.

In all models the bulge and disk potentials are represented by the expressions from Miyamoto and Nagai (1975). The halo component is described by a logarithmic Binney (1981) potential in model IV, is represented by a Plummer (1911) sphere in model V, and is described by a Hernquist (1990) potential in model VI. We solved the fitting problem by taking into account the additional constraints imposed on (a) the local matter density $\rho_{\odot}$ and (b) the force $K_{z=1.1}$ acting perpendicularly to the Galactic plane. As a result, we obtained the model potentials that described a stellar system consistent with the physical characteristics of visible matter in the Galaxy known from observations.

Model VI looks best among the three models considered in this paper from the viewpoint of the accuracy of fitting the model rotation curves to the measurements and to the local matter density and the force acting perpendicularly to the Galactic plane. At the same time, the Navarro-Frenk-White model III is the best one among the six models we considered, the first three of which were considered in our previous paper (Bajkova and Bobylev 2016). It should be noted that models III and VI are close, which we showed, in particular, using the integration of the orbits of two globular clusters as an example.

The Galactic masses within a sphere of radius $50 \mathrm{kpc}$ are close in models IV-VI or, more specifically, $M_{50}=(0.409 \pm 0.020) \times 10^{12} M_{\odot}$ in model IV and $M_{50}=(0.417 \pm 0.034) \times 10^{12} M_{\odot}$ in models $\mathrm{V}$ and VI. The differences between the models increase with increasing radius of the sphere. For example, the Galactic mass within a sphere of radius $200 \mathrm{kpc}$ is $M_{200}=$ $(1.395 \pm 0.082) \times 10^{12} M_{\odot}$ in model IV,$M_{200}=(0.469 \pm 0.038) \times 10^{12} M_{\odot}$ in model $\mathrm{V}$, and $M_{200}=(0.641 \pm 0.049) \times 10^{12} M_{\odot}$ in model VI.

This work was supported by the "Transient and Explosive Processes in Astrophysics" Program P-7 of the Presidium of the Russian Academy of Sciences.

\section{REFERENCES}

1. C. Allen and A. Santillán, Rev.Mex. Astron. Astrofis. 22, 255 (1991).

2. C.A.L. Bailer-Jones, Astron. Astrophys. 575, 35 (2015).

3. A.T. Bajkova and V.V. Bobylev, Astron. Lett. 42, 567 (2016).

4. P. Bhattacharjee, S. Chaudhury, and S. Kundu, Astrophys. J. 785, 63 (2014).

5. J. Binney, Mon. Not. R. Astron. Soc. 196, 455 (1981).

6. V.V. Bobylev and A.T. Bajkova, Astron. Lett. 39, 809 (2013).

7. R.A. Burns, H. Imai, T. Handa, T. Omodaka, A. Nakagawa, T. Nagayama, and Y. Ueno, Mon. Not. R. Astron. Soc. 453, 3163 (2015).

8. W.B. Burton and M.A. Gordon, Astron. Astrophys. 63, 7 (1978).

9. M.A. Butenko and A.V. Khoperskov, Vestn. Volgogr. Univ., Ser. 1: Mat. Fiz., No. 1 (20), 61 (2014).

10. R. Capuzzo-Dolcetta and G. Fragione, Mon. Not. R. Astron. Soc. 454, 2677 (2015). 
11. Y.K. Choi, K. Hachisuka, M.J. Reid, Y. Xu, A. Brunthaler, K.M. Menten, and T.M. Dame, Astrophys. J. 790, 99 (2014).

12. B. Dauphole and J. Colin, Astron. Astrophys. 300, 117 (1995).

13. A.J. Deason, V. Belokurov, N.W. Evans, and J. An, Mon. Not. R. Astron. Soc. 424, L44 (2012a).

14. A.J. Deason, V. Belokurov, N.W. Evans, S.E. Koposov, R.J. Cooke, J. Penarrubia, C.F.P. Laporte, M. Fellhauer, et al., Mon. Not. R. Astron. Soc. 425, 2840 (2012b).

15. G.N. Dremova, V.V. Dremov, V.V. Orlov, A.V. Tutukov, and K.S. Shirokova, Astron Rep. 59, 1019 (2015).

16. G.M. Eadie, W.E. Harris, and L.M. Widrow, Astrophys. J. 806, 54 (2015).

17. N.W. Evans, Mon. Not. R. Astron. Soc. 260, 191 (1993).

18. M. Fellhauer, V. Belokurov, N.W. Evans, M.I. Wilkinson, D.B. Zucker, G. Gilmore, M.J. Irwin, D.M. Bramich, et al., Astrophys. J. 651, 167 (2006).

19. O.Y. Gnedin, W.R. Brown, M.J. Geller, and S.J. Kenyon, Astrophys. J. 720, L108 (2010).

20. G.A. Gontcharov, A.T. Bajkova, P.N. Fedorov, and V.S. Akhmetov, Mon. Not. R. Astron. Soc. 413, 1581 (2011).

21. K. Hachisuka, Y.K. Choi, M.J. Reid, A. Brunthaler, K.M. Menten, A. Sanna, and T.M. Dame, Astrophys. J. 800, 2 (2015).

22. L. Hernquist, Astrophys. J. 356, 359 (1990).

23. A. Irrgang, B. Wilcox, E. Tucker, and L. Schiefelbein, Astron. Astrophys. 549, 137 (2013).

24. A. Just, P. Berczik,M. I. Petrov, and A. Ernst, Mon. Not. R. Astron. Soc. 392, 969 (2009).

25. J.S. Kalirai, J. Anderson, H.B. Richer, I.R. King, J.P. Brewer, G. Carraro, S.D. Davis, G.G. Fahlman, B.M.S. Hansen, et al., Astrophys. J. 657, L93 (2007).

26. I.D. Karachentsev, O.G. Kashibadze, D.I. Makarov, and R.B. Tully, Mon.Not. R. Astron. Soc. 393, 1265 (2009).

27. N.V. Kharchenko, A.E. Piskunov, E. Schilbach, S. Roeser, and R.-D. Scholz, Astron. Astrophys. 558, A53 (2013).

28. G.G. Kuzmin, Astron. Zh. 33, 27 (1956).

29. M. Miyamoto and R. Nagai, Publ. Astron. Soc. Jpn. 27, 533 (1975).

30. K. Motogi, K. Sorai, M. Honma, T. Hirota, K. Hachisuka, K. Niinuma, K. Sugiyama, Y. Yonekura, and K. Fujisawa, Publ. Astron. Soc. Jpn. 68, 69 (2016).

31. J.F. Navarro, C.S. Frenk, and S.D.M. White, Astrophys. J. 490, 493 (1997).

32. H.C. Plummer, Mon. Not. R. Astron. Soc. 71, 460 (1911).

33. M.J. Reid, K.M. Menten, A. Brunthaler, X.W. Zheng, T.M. Dame, Y. Xu, Y. Wu, B. Zhang, et al., Astrophys. J. 783, 130 (2014).

34. A. Ružička, C. Theis, and J. Palouš, Astrophys. J. 725, 369 (2010).

35. M. Sato, Y.W. Wu, K. Immer, B. Zhang, A. Sanna, M.J. Reid, T.M. Dame, A. Brunthaler, and K.M. Menten, Astrophys. J. 793, 72 (2014).

36. D. Veras and N.W. Evans, Mon. Not. R. Astron. Soc. 430, 403 (2013).

37. M.I. Wilkinson and N.W. Evans, Mon. Not. R. Astron. Soc. 310, 645 (1999).

38. A.A. Williams and N.W. Evans, Mon. Not. R. Astron. Soc. 454, 698 (2015).

39. Y.W. Wu, M. Sato, M.J. Reid, L. Moscadelli, B. Zhang, Y. Xu, A. Brunthaler, K.M. Menten, T.M. Dame, and X.W. Zheng, Astron. Astrophys. 566, 17 (2014).

40. A.V. Zasov and K.A. Postnov, General Astrophysics (Vek 2, Fryazino, 2006) [in Russian]. 